\title{
Mathematically arterialised venous blood is a stable representation of patient acid-base status at steady state following acute transient changes in ventilation
}

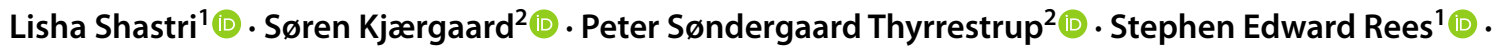 \\ Lars Pilegaard Thomsen ${ }^{1}$
}

Received: 17 May 2021 / Accepted: 5 October 2021 / Published online: 13 October 2021

(c) The Author(s), under exclusive licence to Springer Nature B.V. 2021

\begin{abstract}
Hyper- or hypoventilation are commonly occurring stress responses to arterial puncture around the time of blood sampling and have been shown to rapidly alter arterial blood acid-base parameters. This study aimed to evaluate a physiology-based mathematical method to transform peripheral venous blood acid-base values into mathematically arterialised equivalents following acute, transient changes in ventilation. Data from thirty patients scheduled for elective surgery were analysed using the physiology-based method. These data described ventilator changes simulating 'hyper-' or 'hypoventilation' at arterial puncture and included acid-base status from simultaneously drawn blood samples from arterial and peripheral venous catheters at baseline and following ventilatory change. Venous blood was used to calculate mathematically arterialised equivalents using the physiology-based method; baseline values were analysed using Bland-Altman plots. When compared to baseline, measured arterial and calculated arterialised values at each time point within limits of $\mathrm{pH}: \pm 0.03$ and $\mathrm{PCO}_{2}: \pm 0.5 \mathrm{kPa}$, were considered 'not different from baseline'. Percentage of values considered not different from baseline were calculated at each sampling timepoint following hyper- and hypoventilation. For the physiological method, bias and limits of agreement for $\mathrm{pH}$ and $\mathrm{PCO}_{2}$ were $-0.001(-0.022$ to 0.020$)$ and $-0.02(-0.37$ to 0.33$) \mathrm{kPa}$ at baseline, respectively. $60 \mathrm{~s}$ following a change in ventilation, $100 \%$ of the mathematically arterialised values of $\mathrm{pH}$ and $\mathrm{PCO}_{2}$ were not different from baseline, compared to less than $40 \%$ of the measured arterial values at the same timepoint. In clinical situations where transient breath-holding or hyperventilation may compromise the accuracy of arterial blood samples, arterialised venous blood is a stable representative of steady state arterial blood.
\end{abstract}

Keywords Acid-base $\cdot$ Blood gas analysis $\cdot$ Arterial $\cdot$ Mathematical model $\cdot$ Arterialisation $\cdot$ Hyperventilation $\cdot$ Hypoventilation

\section{Introduction}

Blood gases are an important tool to assess the acid-base status of blood, where values from arterial blood are considered to be the reference. In recent years, venous blood has been used instead of arterial blood for assessment of

Lisha Shastri

lisha@hst.aau.dk

1 Respiratory and Critical Care (RCare) Group, Aalborg University, Fredrik Bajers Vej 7E, 9220 Aalborg Øst, Denmark

2 Department of Anaesthesiology and Intensive Care, Aalborg University Hospital North, Aalborg, Denmark acid-base status $[1,2]$. However, there has been criticism on the usefulness of values from venous blood as a direct substitute for arterial blood gases [3]. Many studies have shown that although venous blood $\mathrm{pH}$ has an acceptable agreement with arterial blood $\mathrm{pH}$, the agreement with $\mathrm{PCO}_{2}$ is poor [2-6], this being particularly true for values of $\mathrm{PCO}_{2}$ over $4.7 \mathrm{kPa}$ [4]. To improve the applicability of venous blood measurements, a method has been developed to calculate 'arterialised' values from venous, i.e., a mathematical arterialisation of venous blood (here after referred to as 'physiological method') [7]. This method applies physiology based mathematical models [8], and has been shown to calculate arterialised values within 2 standard deviations (SDs) of arterial blood values, this being \pm 0.03 for $\mathrm{pH}$ and $\pm 0.5 \mathrm{kPa}$ for $\mathrm{PCO}_{2}$ [9], when evaluated in patients 
treated in departments of emergency [10] or pulmonary medicine [9, 11-13].

When comparing arterialised values to measured arterial values using this physiology-based method, it is often overlooked that inherent variability may exist in arterial measurements. Arterial blood gases (ABGs) are known to exhibit breath-by-breath fluctuations, even in physiological steady state [14], due to the rapid response of arterial blood to changes in ventilation [15]. For monitoring changes in mechanical ventilation, this rapid response can be seen as beneficial in assessing patient state (Fig. 1A). However, where changes in ventilation are transient and reversible, for example a short period of hyperventilation due to fear of arterial puncture, the rapid arterial response may simply add error to understanding the true arterial status without the transient change (Fig. 1B). Fear and anxiety due to the sight of the needle [16], or a response to the pain of the arterial puncture can cause these rapid, transient changes in ventilation, and can be seen as changes in arterial gases within a minute [15, 17-19]. It may therefore be difficult to rely upon measured arterial values under such conditions.

Venous blood however, has been shown to respond more slowly to these changes $[15,18]$. This would make it slower, and hence less useful, than arterial blood in identifying nontransient changes in ventilation such as changes in ventilator settings, seen in Fig. 1A. This slower response does, however, dampen the rapid fluctuations due to transient changes in ventilation seen in Fig. 1B. In situations of acute voluntary or unstable ventilation, it is therefore possible that arterialised values from a peripheral venous blood sample, using the physiology-based method [7], could be a more stable description of patient status at steady state than measured
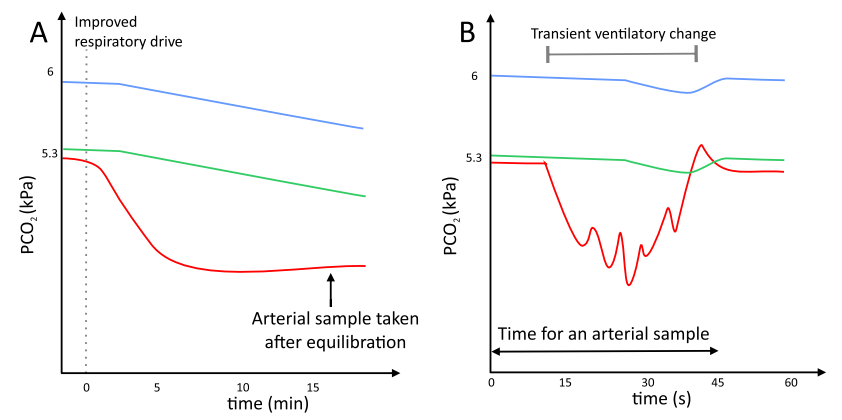

Fig. 1 Graphical illustrations of the concept of a transient ventilatory change A: Illustrates the clinical setting where guidelines recommend waiting for at least $10 \mathrm{~min}$ for equilibration of blood gases before drawing an arterial sample. Here the arterial blood most accurately reflects the blood gases intending to be measured. B: Illustrates the changes that can be seen in response to a transient change in ventilation. The figure shows how the arterial blood (red) responds rapidly to the changes in ventilation, while the venous (blue) and hence arterialised blood (green) remains stable for the duration of the blood sampling, thereby better reflecting the steady-state values that were intended to be measured. (Color figure online) arterial values, effectively filtering out the effects of transient changes in ventilation.

This study investigates use of the physiology-based method to remove the effects of acute, transient changes in ventilation on arterial blood acid-base values. It is hypothesised that arterial values prior to the onset of transient changes in ventilation can be accurately calculated with the method, and that unlike measured arterial values, calculated arterialised values remain stable during transient ventilatory changes.

\section{Methods}

\subsection{Patients}

Data for this study were collected as part of a protocol, detailed in a previous publication [15]. Patients without cardiovascular or respiratory disease, scheduled for elective robotic assisted laparoscopic prostatectomy or hysterectomy, were studied, thereby allowing for the isolation of ventilatory changes without the presence of metabolic and perfusion disturbances. The study was approved by the Regional Ethical Committee for North Jutland and abides by the guidelines set in the Declaration of Helsinki.

\subsection{Physiology-based method}

Figure 2 outlines the principle behind the physiology based mathematical method for arterialising venous blood [7]. The principle of the method is that peripheral venous blood is mathematically transported back through the tissues adding and removing oxygen and carbon dioxide in a fixed ratio until oxygen levels match measurements taken from the pulse oximeter. The steps of this process are as follows and illustrated in Fig. 2: Venous blood values are first measured in a blood gas analyser (Step ' $a$ '), following which the total content of blood gases $\left(\mathrm{tO}_{2}\right.$ and $\left.\mathrm{tCO}_{2}\right)$ is calculated (Step 'b'). To transform these into arterialised equivalents, the oxygen concentration difference $\left(\Delta \mathrm{O}_{2}\right)$ between arterial and venous blood is estimated (Step ' $c$ '). This is done using venous haemoglobin $\left(\mathrm{Hb}_{\mathrm{v}}\right)$ and $\mathrm{SvO}_{2}$ for venous blood, and using $\mathrm{Hb}_{\mathrm{v}}$ and $\mathrm{SpO}_{2}$ for arterial blood. With the assumption of a respiratory quotient (RQ) of 0.82 , representing the average aerobic metabolism in the tissues, and considering the Bohr and Haldane effects, arterial $\mathrm{CO}_{2}$ concentration is calculated as detailed in Fig. 2 Step 'c'. The model assumes negligible acid addition or removal across the tissues, or anaerobic metabolism, and simulates the reverse transport of blood from veins to arteries, which allows for the calculation of 'arterialised' equivalents (Step 'd'). The value of $\Delta \mathrm{O}_{2}$ is varied until the difference between arterialised oxygen 


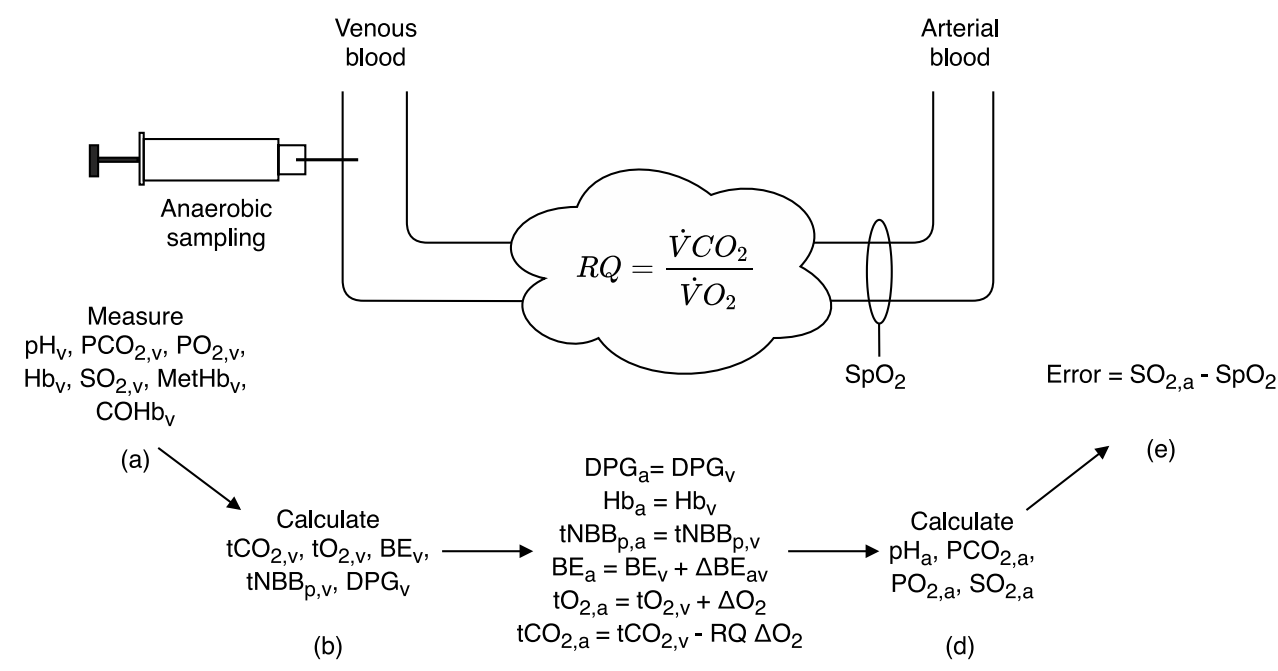

(c)

Fig. 2 Physiology based mathematical method from Rees et al. [7]. Figure depicting the principles of the physiology based mathematical method, used to transform venous blood values to arterial equivalents, in 5 steps ('a' to 'e'). Subtexts: a: arterial, v: venous, p: plasma; $\mathrm{PCO}_{2}: \mathrm{CO}_{2}$ tension $(\mathrm{kPa}) ; \mathrm{PO}_{2}: \mathrm{O}_{2}$ tension $(\mathrm{kPa}) ; \mathrm{Hb}$ : haemoglobin $(\mathrm{mmol} / \mathrm{l}) ; \mathrm{SO}_{2}: \mathrm{O}_{2}$ saturation $(\%) ;$ MetHb: Methaemoglobin (\%);

saturation $\left(\mathrm{SO}_{2} \mathrm{a}\right)$ and that measured by the pulse oximeter $\left(\mathrm{SpO}_{2}\right)$ is minimised (Step ' $\mathrm{e}$ ').

\subsection{Protocol}

The study included changes in mechanical ventilation followed by rapid arterial and peripheral venous blood sampling, the details of which are described below. It is important to note that these changes in mechanical ventilation were used to simulate the transient changes in ventilation as illustrated in Fig. 1B. Patients were studied in the operation room, between the onset of anaesthesia and start of surgery. Patients were sedated with total intravenous anaesthesia prior to and for the duration of the study, and arterial and peripheral venous catheters were inserted in the radial artery and cubital vein, respectively, following patient sedation.

\subsubsection{Blood gas sampling}

Pairs of blood samples were taken by two trained individuals, drawing arterial and peripheral venous blood samples, simultaneously, at baseline, and 15, 30, 45, 60, 90 and $120 \mathrm{~s}$ after a change in ventilation. Blood sampling was stopped at $120 \mathrm{~s}$ to reflect the focus of this study, i.e., evaluation of the effects of short-term transient changes (Fig. 1B), rather than changes to steady state (Fig. 1A). Blood samples were collected in pre-heparinised safePICO syringes, which were capped immediately after sampling, and following removal of air bubbles, if any. A third person assisted with
COHb: carboxyhaemoglobin (\%); $\mathrm{tCO}_{2}$ : total $\mathrm{CO}_{2}$ content (mmol/l); $\mathrm{tO}_{2}$ : total $\mathrm{O}_{2}$ content (mmol/l); RQ: respiratory quotient; BE: base excess (mmol/l); $\mathrm{SpO}_{2}$ : peripheral saturation of $\mathrm{O}_{2}$ measured via pulse oximetry $(\%) ; \Delta \mathrm{O}_{2}:$ a-v $\mathrm{O}_{2}$ concentration difference. Figure reproduced with permissions [36]

the capping of the syringes and ensured the precise timing of the sampling. Samples were analysed in the same order as they were taken (with arterial analysed first, followed by the corresponding venous sample), on the ABL800 Flex analyser (Radiometer, Copenhagen, Denmark).

\subsubsection{Ventilatory changes}

Patients were randomised and received changes in mechanical ventilation corresponding to either 'hyperventilation' or 'hypoventilation'. Ventilator settings at baseline were set at a tidal volume $\left(\mathrm{V}_{\mathrm{T}}\right)$ of $6 \mathrm{ml} / \mathrm{kg}$, respiratory rate $(\mathrm{RR})$ of 15 breaths per min (bpm), and PEEP of $5 \mathrm{cmH}_{2} \mathrm{O}$. Changes received by the patients to simulate hyperventilation and hypoventilation were a combination of $\mathrm{V}_{\mathrm{T}}$ and $\mathrm{RR}$, i.e., Hyperventilation: $\mathrm{V}_{\mathrm{T}}=8 \mathrm{ml} / \mathrm{kg}$ and $\mathrm{RR}=20 \mathrm{bpm}$, and hypoventilation: $\mathrm{V}_{\mathrm{T}}=6 \mathrm{ml} / \mathrm{kg}$ and $\mathrm{RR}=6 \mathrm{bpm}$. This change lasted $2 \mathrm{~min}$, thereby characterising typical short-term changes in ventilation. $\mathrm{SpO}_{2}$ was monitored throughout the study using pulse oximetry.

\subsection{Data analysis}

A sample size calculation was performed using previous studies $[17,18]$ showing that 12 patients each for hyperand hypoventilation were needed, and as such it was decided to study 30 patients. Further details are described in [15].

To evaluate whether the physiological method could accurately describe arterial values prior to acute, transient 
changes in ventilation, peripheral venous blood gases and $\mathrm{SpO}_{2}$ values taken at baseline were used to calculate mathematically arterialised equivalents of acid-base status and these compared to measured arterial values using a Bland-Altman comparison.

To evaluate whether mathematically arterialised values remain stable during acute, transient ventilatory changes, arterialised values were calculated from peripheral venous blood for each time point following the change in ventilation. Measured arterial and calculated arterialised values of $\mathrm{pH}$ and $\mathrm{PCO}_{2}$ were then compared against baseline values. These were considered to be clinically equivalent to baseline values if the difference between the values at each sampling time point and their corresponding baseline values, were within pre-defined limits, described below. For each sampling time point following an acute, transient change in ventilation, the percentage of measured arterial and calculated arterialised values, within the pre-defined limits were calculated. This process was performed for the changes in ventilation both due to hyper- and hypoventilation.

The pre-defined limits were selected to reflect previously measured arterial variability between samples taken in quick succession in steady-state, with these having been shown to vary in the range of 0.02 to 0.05 for $\mathrm{pH}$ and between 0.3 to $0.6 \mathrm{kPa}$ for $\mathrm{PCO}_{2}[20,21]$. These limits are consistent with the previously demonstrated efficacy of the physiological method to transform peripheral venous blood to arterialised equivalents, i.e., the calculated arterialised ('ca') to measured arterial ('a') difference in the range of \pm 0.03 for $\mathrm{pH}$ and $\pm 0.5 \mathrm{kPa}$ for $\mathrm{PCO}_{2}$ [9]. The pre-defined limits used in this study were for $\mathrm{pH}: \pm 0.03$ and $\mathrm{PCO}_{2}: \pm 0.5 \mathrm{kPa}$, where a difference between values at each time point and their corresponding values at baseline, within these limits were categorised as 'not different from baseline'.

\section{Results}

A total of 30 patients were studied, with 15 each receiving changes of hyper- or hypoventilation. The patients were aged 61 years on average and $73 \%$ were male. Further details of the population are described in Table 1 and in [15].

Figure 3 illustrates the comparison of arterialised values of $\mathrm{pH}$ and $\mathrm{PCO}_{2}$ with arterial measurements at baseline. The physiological method calculated arterialised values with bias and limits of agreement (LoA) for $\mathrm{pH}=-0.001(-0.022$ to $0.020)$ and $\mathrm{PCO}_{2}=-0.02(-0.37$ to 0.33$) \mathrm{kPa}$.

Figure 4 depicts the percentage of measured arterial and calculated arterialised values of $\mathrm{pH}$ and $\mathrm{PCO}_{2}$ within the pre-defined limits of \pm 0.03 and $\pm 0.5 \mathrm{kPa}$, respectively, at each sampling timepoint following hyper- and hypoventilation. $60 \mathrm{~s}$ following a change in ventilation, $100 \%$ of the arterialised values of $\mathrm{pH}$ and $\mathrm{PCO}_{2}$ were within the
Table 1 Population characteristics $(\mathrm{n}=30)$

\begin{tabular}{ll}
\hline Parameters & Mean \pm SD or N (\%) \\
\hline Age (years) & $61 \pm 10$ \\
Sex- Male & $22(73.3)$ \\
Surgery: Prostatectomy & $22(73.3)$ \\
Hysterectomy & $8(26.7)$ \\
Measured arterial (baseline): $\mathrm{pH}$ & $7.409 \pm 0.035$ \\
$\mathrm{PCO}_{2}(\mathrm{kPa})$ & $5.55 \pm 0.64$ \\
$\mathrm{Calculated}$ arterial (baseline): $\mathrm{pH}$ & $7.410 \pm 0.033$ \\
$\mathrm{PCO}_{2}(\mathrm{kPa})$ & $5.58 \pm 0.61$ \\
\hline
\end{tabular}

pre-defined limits, in comparison to less than $40 \%$ of the measured arterial values at the same timepoint. In addition, while nearly all the measured arterial samples were outside the pre-defined limits by the end of the study, more than half of the arterialised samples were still within the limits. As an example, data from a single patient is presented in Fig. 5. Here, measured arterial $\mathrm{pH}$ and $\mathrm{PCO}_{2}$ changed by 0.034 and $0.63 \mathrm{kPa}$ respectively, while calculated values changed by 0.007 and $0.17 \mathrm{kPa}$, at the end of the study when compared to baseline.

\section{Discussion}

Blood gas analysis is the mainstay for monitoring a patient's acid-base status. Arterial blood sampling is considered the reference technique but can be painful and risks multiple complications [22]. Venous blood is a valuable surrogate for arterial blood. However, there is debate regarding its acceptability as a direct substitute for arterial acid-base status. A physiology based mathematical method could bridge this gap, with calculation of arterialised values from peripheral venous blood [7]. This study has illustrated for the first time, that in the event of acute transient changes in ventilation, arterialised values of $\mathrm{pH}$ and $\mathrm{PCO}_{2}$ calculated from peripheral venous blood, remain a stable representation of steady state arterial values, effectively filtering out transient ventilatory disturbances.

As part of setting a baseline to investigate the effect of ventilatory changes, this study has once again established that the physiological method can calculate arterialised values well within the variability of repeated arterial blood measurements at steady state. The physiological method was able to calculate values with 2 SDs of \pm 0.02 for $\mathrm{pH}$ and $\pm 0.35 \mathrm{kPa}$ for $\mathrm{PCO}_{2}$, which were comparable to previous publications by Tygesen et al. [10] reporting 2SDs of \pm 0.024 for $\mathrm{pH}$ and $0.46 \mathrm{kPa}$ for $\mathrm{PCO}_{2}$, and Ekström et al. [11] reporting $2 \mathrm{SDs}$ of \pm 0.016 and $0.33 \mathrm{kPa}$, both examining the physiological method on patients in steady state ventilation. 


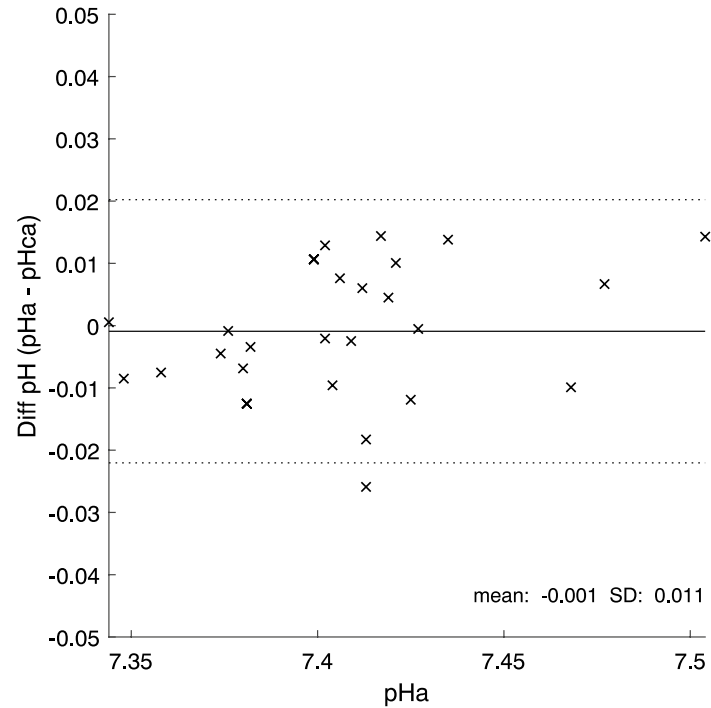

Fig. 3 Bland-Altman comparison of the mathematically arterialised blood against the measured arterial blood at baseline. Bland-Altman plot comparing calculated arterialised ('ca') blood to the measured

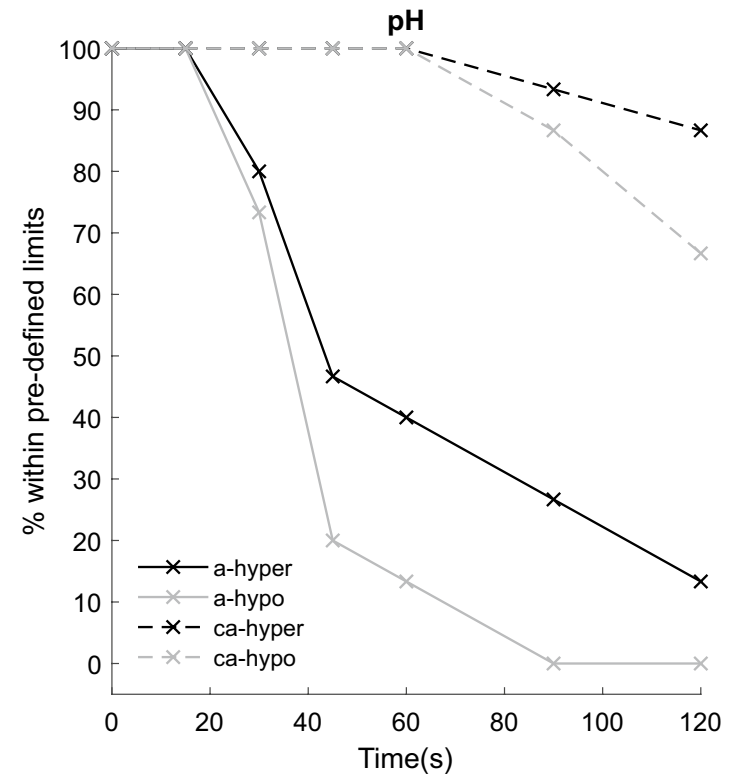

Fig. 4 Percentage of $\mathrm{pH}$ and $\mathrm{PCO}_{2}$ in measured arterial and calculated arterialised blood within pre-defined limits, at each sampling timepoint following acute transient changes in ventilation. Proportion of blood gas samples within the limits of \pm 0.03 for $\mathrm{pH}$ and $\pm 0.5 \mathrm{kPa}$ for $\mathrm{PCO}_{2}$ when measured arterial ('a'; solid lines) and calculated

In addition, the results from this study demonstrate that the physiological method can calculate arterialised values of $\mathrm{pH}$ and $\mathrm{PCO}_{2}$ very close to the baseline measured arterial values, with $100 \%$ of samples after $1 \mathrm{~min}$, and over $60 \%$ of samples at the end of 2 min within the pre-defined acceptable limits following acute transient changes in ventilation.

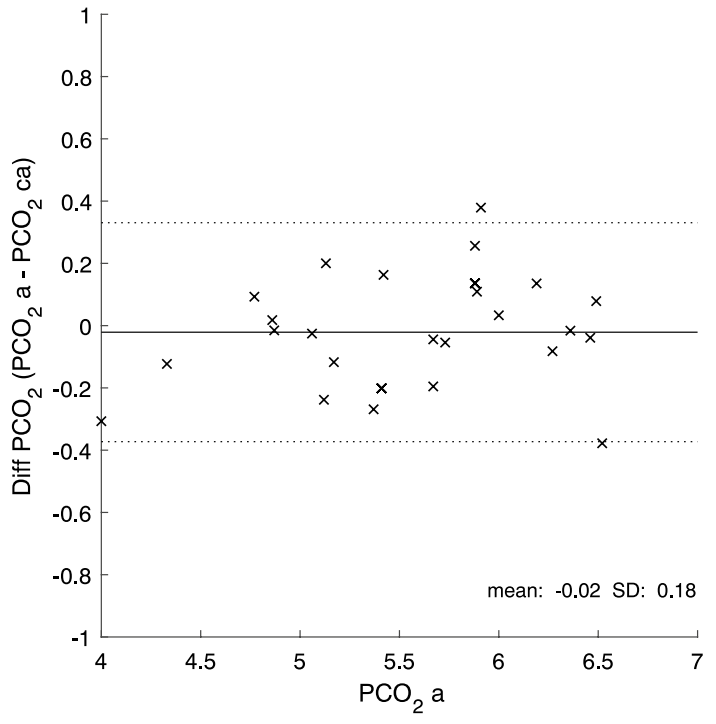

arterial ('a') blood at baseline, plotted against the measured arterial on the $\mathrm{x}$-axis. $\mathrm{N}=30$. Bias (solid line) and LoAs (dotted lines) are displayed

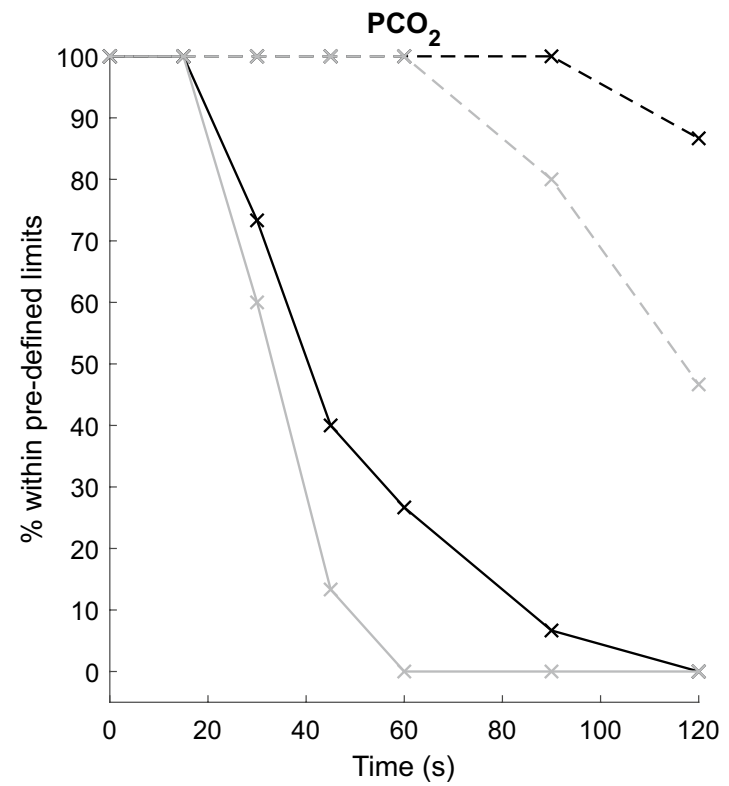

arterialised ('ca'; dashed lines) values at each sampling timepoint are compared to their respective baseline values, in the 2 min following a simulated acute transient change in ventilation (hyperventilation (black) or hypoventilation (grey), $\mathrm{N}=15$ each)

This is in contrast to the measured arterial values which changed over thrice as much as the arterialised values, beginning as early as $30 \mathrm{~s}$ following a change in ventilation. There are potential clinical consequences of transient changes in ventilation around the point of sampling due to reversible breath holding or hyperventilation caused by fear 
Fig. 5 Example of a single patient's blood acid-base response to acute simulated hyperventilation.Figure illustrating changes $(\Delta)$ in $\mathrm{pH}$ and $\mathrm{PCO}_{2}$ from baseline in measured arterial ('a'; red) and calculated arterialised ('ca'; black) blood following acute simulated hyperventilation. (Color figure online)

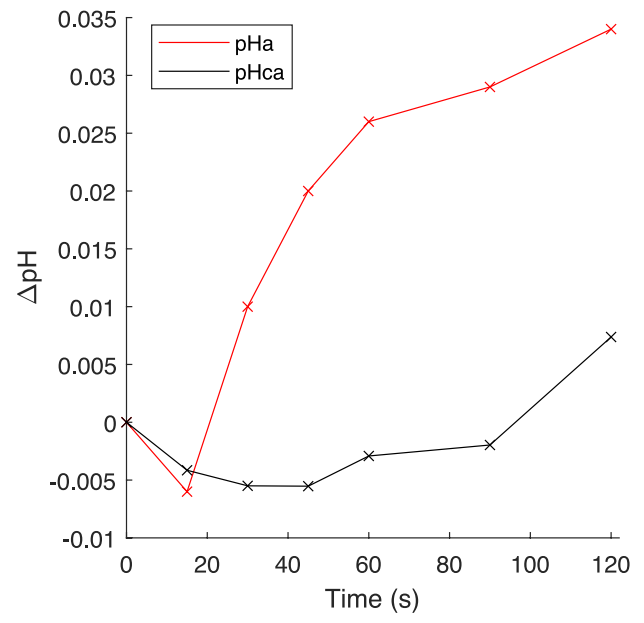

of arterial puncture. The rapid response of arterial blood to these transient changes in ventilation, could indeed increase the risk of misinterpretation of patient state while using the $\mathrm{ABG}$ results during for example, titration of non-invasive ventilation settings [23], initiation of long term oxygen therapy for chronic obstructive pulmonary disease (COPD) patients [24], or clinical decisions for patients presenting to the emergency department [25] amongst other situations. As an example, for a patient diagnosed with COPD, guidelines suggest different treatment options based on whether $\mathrm{pH}$ is below 7.35 or $\mathrm{PCO}_{2}$ is above $6.7 \mathrm{kPa}$ [26]. If a patient's arterial $\mathrm{pH}$ and $\mathrm{PCO}_{2}$ were measured to be 7.35 and $6.7 \mathrm{kPa}$, it could be possible for the patient's real steady state values to be anywhere between 7.316-7.384 and $6.07-7.33 \mathrm{kPa}$ if values were measured during transient changes in ventilation. While these values reflect those taken from Fig. 4 above, in reality, changes in ventilation could range from hyperventilating at frequencies of over $30 \mathrm{bpm}$, to varying durations of breath-holding, representing complete apnoea, disturbances much greater than those studied here. The degree of these responses initiated by transient ventilatory disturbances, could affect the decisions and treatments in the sequence of patient management. Although changes can vary widely between patients, the speed at which arterial blood responds to these changes would still be comparable to those seen in this study. A trained individual has been shown to take between $30-60 \mathrm{~s}$ to perform an arterial puncture [27], and it is therefore likely that changes in ventilation could affect the arterial blood being sampled within this period.

A further factor resulting in transient changes in ventilation is the fear and anxiety caused by arterial punctures [16, 28]. Kose et al. [28] reported around $27 \%$ of adults displayed symptoms of anxiety and shortness of breath in response to receiving an injection or having blood drawn. In addition, fear of the needle can also lead to patients avoiding or delaying medical procedures or treatment [16] resulting in an increase in patient numbers and costs to the public healthcare sector. Many hospitals have evaluated ways to decrease the number of ABGs taken, by introduction of protocols based on specific indications rather than daily routine [29-32], especially in the intensive care units, thereby reducing the load on the hospital staff, equipment and costs. However, this does not decrease the pain and patient discomfort associated with an arterial puncture [33], or the blood loss and catheter infections associated with an arterial line $[31,34]$. In this regard, venous blood has been suggested as an alternative, however, venous blood values alone proving inadequate for clinical decision making as no guidelines exist allowing for its direct application. In contrast, the physiological method allows for the calculation of arterialised values which could be used in place of the measured arterial parameters and are therefore applicable in the use of treatment guidelines.

\section{Limitations}

The major limitation of this study was that the response of the arterial and venous blood after returning the ventilatory settings to baseline was not studied. Immediate return to baseline would have been appropriate to simulate the clinical situation of transient, reversible, changes in ventilation, i.e., the focus of this study. The number of samples were limited to those obtained within $2 \mathrm{~min}$ so as to maintain the quality of the samples, where we were able to analyse the samples on the same blood gas analyser within 20-25 min, as suggested by the IFCC guidelines [35]. In addition, this study has not investigated the time response to a new steady state of the venous blood, and conclusions cannot therefore be drawn as to the use of the physiological method to measure patient response to, for example, changes in mechanical ventilation. It is likely that the delayed response illustrated here would require a longer time to equilibrium for venous blood than the usual 10-15 min for arterial blood. 


\section{Conclusion}

In conclusion, this study reaffirms the reliability of the physiology based mathematical model for transformation of venous blood acid-base status to arterialised equivalents. In addition, it also shows that arterialised venous blood is a more stable representative of steady state arterial blood gas values following acute, transient changes in ventilation.

Authors' contributions LS, LPT, SK and SER conceptualised the study. LS, LPT, PST conducted the study. LS, LPT and SER analysed the data, and all authors contributed to the interpretation of the results and writing of the manuscript. All authors read and approved the final manuscript.

Funding The research group of LS, SER and LPT receive research money from OBI Medical A/S.

Data availability The datasets used and/or analysed during the current study are available from the corresponding author on reasonable request.

Code availability Not Applicable.

\section{Declarations}

Conflict of interest SER and SK were previous shareholders of OBI Medical A/S.

Ethical approval The study was approved by the Regional Ethical Committee for North Jutland (N-20190014) and abides by the guidelines set in the Declaration of Helsinki.

Consent to participate Written informed consent was obtained from all individual participants included in the study.

Consent for publication Not applicable.

\section{References}

1. Kelly AM. Can venous blood gas analysis replace arterial in emergency medical care. Emerg Med Australas. 2010;22:493-8.

2. Bloom BM, Grundlingh J, Bestwick JP, Harris T. The role of venous blood gas in the emergency department. Eur J Emerg Med. 2014;21:81-8.

3. Byrne AL, Bennett M, Chatterji R, Symons R, Pace NL, Thomas PS. Peripheral venous and arterial blood gas analysis in adults: are they comparable? A systematic review and meta-analysis. Respirology. 2014;19:168-75.

4. Nanjayya VB, Mccracken P, Vallance S, Board J, Kelly PJ, Schneider HG, et al. Arterio-VENouS intra subject agreement for blood gases within intensive care: the AVENSIS study. J Intensive Care Soc. 2020;21:64-71.

5. McKeever TM, Hearson G, Housley G, Reynolds C, Kinnear W, Harrison TW, et al. Using venous blood gas analysis in the assessment of COPD exacerbations: a prospective cohort study. Thorax. 2016;71:210-5.
6. Malatesha G, Singh NK, Bharija A, Rehani B, Goel A. Comparison of arterial and venous $\mathrm{pH}$, bicarbonate, $\mathrm{PCO} 2$ and $\mathrm{PO} 2$ in initial emergency department assessment. Emerg Med J. 2007;24:569-71.

7. Rees SE, Toftegaard M, Andreassen S. A method for calculation of arterial acid-base and blood gas status from measurements in the peripheral venous blood. Comput Methods Programs Biomed. 2006;81:18-25.

8. Rees SE, Andreassen S. Mathematical models of oxygen and carbon dixide storage and transport: the acid-base chemistry of blood. Crit Rev Biomed Eng. 2005;33:209-64.

9. Rees SE, Hansen A, Toftegaard M, Pedersen J, Kristiensen SR, Harving H. Converting venous acid-base and oxygen status to arterial in patients with lung disease. Eur Respir J. 2009;33:1141-7.

10. Tygesen G, Matzen H, Grønkjær K, Uhrenfeldt L, Andreassen S, Gaardboe $\mathrm{O}$, et al. Mathematical arterialization of venous blood in emergency medicine patients. Eur J Emerg Med. 2012;19:363-72.

11. Ekström M, Engblom A, Ilic A, Holthius N, Nordström P, Vaara I. Calculated arterial blood gas values from a venous sample and pulse oximetry: clinical validation. PLoS One. 2019;14:e0215413.

12. Thomsen LP, Klein A-C, Vitali-Serdoz L, Bastian D, Shastri L, Rees SE, et al. Evaluation of mathematical arterialization of venous blood in intensive care and pulmonary ward patients. Respiration. 2021;100:1-9.

13. Rees SE, Rychwicka-Kielek BA, Andersen BF, Bibi R, Pedersen $\mathrm{JF}$, Weinreich UM, et al. Calculating acid-base and oxygenation status during COPD exacerbation using mathematically arterialised venous blood. Clin Chem Lab Med. 2012;50:2149-54.

14. Formenti F, Bommakanti N, Chen R, Cronin JN, McPeak H, Holopherne-Doran D, et al. Respiratory oscillations in alveolar oxygen tension measured in arterial blood. Sci Rep. 2017;7:1-10.

15. Shastri L, Kjærgaard S, Thyrrestrup PS, Rees SE, Thomsen LP. Is venous blood a more reliable description of acid-base state following simulated hypo- and hyperventilation? Scand J Trauma Resusc Emerg Med. 2021;29:35.

16. McLenon J, Rogers MAM. The fear of needles: a systematic review and meta-analysis. J Adv Nurs. 2019;75:30-42.

17. Steurer J, Hoffmann U, Dür P, Russi E, Vetter W. Changes in arterial and transcutaneous oxygen and carbon dioxide tensions during and after voluntary hyperventilation. Respiration. 1997;64:200-5.

18. Montagna P, Paniccia M, Marchello L, Riva R. Hyperventilation in normal subjects. A clinical, gas-analytic and EMG study. Electroencephalogr Clin Neurophysiol. 1995;97:159-63.

19. Sasse SA, Berry RB, Nguyen TK, Light RW, Kees MC. Arterial blood gas changes during breath-holding from functional residual capacity. Chest Elsevier. 1996;110:958-64.

20. Thorson SH, Marini JJ, Pierson DJ, Hudson LD. Variability of arterial blood gas values in stable patients in the ICU. Chest. 1983;84:14-8.

21. Mallat J, Lazkani A, Lemyze M, Pepy F, Meddour M, Gasan $\mathrm{G}$, et al. Repeatability of blood gas parameters, PCO2 Gap, and $\mathrm{PCO} 2$ gap to arterial-to-venous oxygen content difference in critically ill adult patients. Medicine. 2015;94:e415.

22. Giner J, Casan P, Belda J, González M, Ma Miralda R, Sanchis J. Pain during arterial puncture. Chest. 1996;110:1443-5.

23. Davidson C, Banham S, Elliot M, Kennedy D, Gelder C, Glossop A, et al. BTS/ICS guidelines for the ventilatory management of acute hypercapnic respiratory failure in adults. Thorax. 2016;71:1-35.

24. Hardinge M, Annandale J, Bourne S, Cooper B, Evans A, Freeman D, et al. British thoracic society guidelines for home oxygen use in adults. Thorax. 2015;70:1-43.

25. Raffin TA. Indications for arterial blood gas analysis. Ann Intern Med. 1986;105:390.

26. Celli BR, Macnee W, Agusti A, Anzueto A, Berg B, Buist AS, et al. Standards for the diagnosis and treatment of patients with 
COPD: a summary of the ATS/ERS position paper. Eur Respir J. 2004;23:932-46.

27. Patout M, Lamia B, Lhuillier E, Molano L. A randomized controlled trial on the effect of needle gauge on the pain and anxiety experienced during radial arterial puncture. PLoS One. 2015;10:e0139432.

28. Kose S, Mandiracioglu A. Fear of blood/injection in healthy and unhealthy adults admitted to a teaching hospital. Int J Clin Pract. 2007;61:453-7.

29. Browning JA, Kaiser DL, Durbin CG. The effect of guidelines on the appropriate use of arterial blood gas analysis in the intensive care unit. Respir Care. 1989;34:269-76.

30. DellaVolpe JD, Chakraborti C, Cerreta K, Romero CJ, Firestein $\mathrm{CE}$, Myers L, et al. Effects of implementing a protocol for arterial blood gas use on ordering practices and diagnostic yield. Healthcare Elsevier. 2014;2:130-5.

31. Muakkassa F, Rutledge R, Fakhry S, Meyer A, Sheldon G. $A B G s$ and arterial lines: the relationship to unnecessarily drawn arterial blood gas samples. J Trauma Inj Infect Crit Care. 1990;30:1087-95.

32. Merlani P, Garnerin P, Diby M, Ferring M, Ricou B. Linking guideline to regular feedback to increase appropriate requests for clinical tests: Blood gas analysis in intensive care. Br Med J. 2001;323:620-4.
33. Matheson L, Stephenson M, Huber B. Reducing pain associated with arterial punctures for blood gas analysis. Pain Manag Nurs. 2014;15:619-24.

34. Gershengorn HB, Wunsch H, Scales DC, Zarychanski R, Rubenfeld G, Garland A. Association between arterial catheter use and hospital mortality in intensive care units. JAMA Intern Med American Medical Association. 2014;174:1746-54.

35. Burnett RW, Covington AK, Fogh-Andersen N, Külpmann WR, Maas AHJ, Müller-Plathe O, Siggaard-Andersen O, Van Kessel AL, Wimberley PD, Zijlstra WG. Approved IFCC recommendations on whole blood sampling, transport and storage for simultaneous determination of $\mathrm{pH}$, blood gases and electrolytes. Eur J Clin Chem Clin Biochem. 1995;33:247-53.

36. Shastri L, Boulain T, Rees SE, Thomsen LP. Comparison of two methods for converting central venous values of acid-base status to arterial values in critically ill patients. Comput Methods Programs Biomed. 2021;203:e106022.

Publisher's Note Springer Nature remains neutral with regard to jurisdictional claims in published maps and institutional affiliations. 